\title{
Impact of Simultaneous Versus Sequential Initiation of Basal Insulin and Glucagon-like Peptide-1 Receptor Agonists on HbA1c in Type 2 Diabetes: A Retrospective Observational Study
}

\author{
Xuejun Victor Peng - Rajeev Ayyagari - Robert Lubwama • \\ Lizheng Shi • Eboni G. Price-Haywood • Priscilla Hollander • \\ Vivian Fonseca
}

Received: December 20, 2019 / Published online: February 28, 2020

(C) The Author(s) 2020

\begin{abstract}
Introduction: When and how to intensify treatment in patients with type 2 diabetes (T2D) not achieving glycated hemoglobin (HbA1c) targets with oral antidiabetic drugs (OADs) in clinical practice remains a matter of clinical preference. This pilot study was conducted using the retrospective observational data from such patients to evaluate the impact on HbA1c of three treatment sequences: simultaneous initiation of basal insulin (BI) and a glucagonlike peptide-1 receptor agonist (GLP-1 RA;
\end{abstract}

Enhanced Digital Features To view enhanced digital features for this article go to https://doi.org/10.6084/ m9.figshare.11800605.

X. V. Peng · R. Lubwama

Sanofi US, Inc., 55 Corporate Dr., Bridgewater, NJ, USA

R. Ayyagari

Analysis Group, Inc., 111 Huntington Ave., 14th

Floor, Boston, MA, USA

L. Shi · V. Fonseca ( $₫)$

Tulane University, 1430 Tulane Ave.,

New Orleans, LA, USA

e-mail: vfonseca@tulane.edu

E. G. Price-Haywood

Ochsner Health System, 1514 Jefferson Hwy.,

New Orleans, LA, USA

P. Hollander

Baylor Endocrine Center, Wadley Tower,

3600 Gaston Ave \#656, Dallas, TX, USA
Cohort 1); BI followed by GLP-1 RA initiation within a 90-day timeframe (Cohort 2); or BI followed by GLP-1 RA initiation beyond 90 days (Cohort 3).

Methods: Data from the regional US electronic medical records database, Research Action for Health Network (REACHnet), were extracted for all patients with $\mathrm{T} 2 \mathrm{D}$ aged $\geq 18$ years who had encounter dates between January 2011 and August 2017 and $\geq 1$ HbA1c laboratory value(s) $<90$ days before BI initiation and $\geq 2$ HbA1c laboratory values within 1 year after BI initiation and who met the inclusion criteria for GLP-1 RA initiation set for Cohorts 1, 2, or 3 . The primary endpoints were the proportion of patients achieving $\mathrm{HbA} 1 \mathrm{c}<7.0 \%$, which was estimated via Kaplan-Meier analysis, and change in HbA1c within 12 months.

Results: Overall, 869 patients were analyzed, of whom 109 were in Cohort 1, 301 in Cohort 2, and 459 in Cohort 3. Baseline HbA1c was $10.3 \pm 2.1, \quad 10.3 \pm 2.0$, and $10.2 \pm 2.1 \%$ for these three cohorts, respectively. Statistically significantly more patients in Cohort 1 than in Cohort 3 achieved HbA1c $<7.0 \%$ (33.4 vs. 20.9\%, respectively; $p=0.0186)$. Mean observed reductions in $\mathrm{HbA1c}$ at 12 months were $-1.7 \%$ (Cohort 1$),-1.5 \%$ (Cohort 2), and $-1.3 \%$ (Cohort 3 ).

Conclusions: Simultaneous initiation of BI and GLP-1 RA achieves glycemic control more effectively than sequential initiation of BI with GLP-1 RA added beyond 90 days. 
Keywords: Basal insulin; Glucagon-like peptide-1 receptor agonist; HbA1c; Hyperglycemia; Real-world evidence; Type 2 diabetes mellitus

\section{Key Summary Points}

\section{Why carry out this study?}

A large proportion of US patients with type 2 diabetes (T2D) have inadequate glycemic control.

A basal insulin (BI) in combination with a glucagon-like peptide-1 receptor agonist (GLP-1 RA) is highly effective in reducing glycated hemoglobin (HbA1c).

Is simultaneous initiation of BI and a GLP-1 RA more effective at reducing HbA1c levels than sequential introduction of these injectables as recommended by societal guidelines?

\section{What was learned from the study?}

In clinical practice, as reflected in this US regional database, patients with $\mathrm{T} 2 \mathrm{D}$ are initiated onto BI and GLP-1 RA therapies at very high levels of HbA1c and high body mass index.

Among patients with T2D inadequately controlled on oral antidiabetic medications, simultaneous initiation of BI and a GLP-1 RA resulted in significantly better glycemic control than sequential initiation of BI and GLP-1 RA with a between-treatment gap of $>90$ days.

The longer the gap between the initiation of BI and that of GLP-1 RA, the poorer the glycemic control outcome.

\section{INTRODUCTION}

In the USA, nearly $50 \%$ of patients with type 2 diabetes (T2D) are living with uncontrolled hyperglycemia [1, 2], despite the introduction of numerous new diabetes medications over the past two decades [3, 4]. A retrospective analysis of US claims data from 1.66 million patients with T2D showed a declining trend (from $56.4 \%$ in 2006 to $54.2 \%$ in 2013; $p<0.001$ ) in patients achieving a glycated hemoglobin (HbA1c) target of $<7.0 \%$. This was despite the increase in choice of medications and a significant decrease in the proportion of patients not taking any glucose-lowering agents over the timeframe. Furthermore, the use of potent injectable agents, including insulin and glucagon-like peptide-1 receptor agonists (GLP-1 RAs), has significantly increased [5], suggesting that improvements in the timing and approach of initiation and intensification of these therapies are required to optimize and sustain glycemic control $[6,7]$, particularly given the progressive nature of T2D [8].

Over the past decade, in line with increasing treatment options, guidelines on when and how to initiate and intensify treatments in T2D have continued to evolve $[8,9]$. Back in 2009, the American Diabetes Association/European Association for the Study of Diabetes (ADA/EASD) consensus algorithm recommended-as the preferred treatment pathway for most patients with T2D—the initiation of basal insulin (BI) if glycemic control was inadequate with one or more oral antidiabetic drugs (OADs), with GLP1 RAs recommended in combination with metformin in selected clinical settings only [9]. Following the wealth of clinical data in recent years demonstrating the additional benefits of GLP-1 RAs beyond glycemic control, such as cardiovascular risk reduction and weight loss, the 2019 ADA guidelines further elevated the priority of GLP-1 RAs as the recommended first injectable medication following OADs [2].

Seven of eight pathophysiological defects seen in T2D are addressed by the complementary mechanisms of action of BI and GLP-1 RAs [10], and combination therapy with BI and a GLP-1 RA is highly effective in reducing HbA1c $[2,11,12]$, as well as offering weight neutrality or weight loss, reduced hypoglycemia risk compared with BI alone, and a reduced insulin requirement [13]. These observations have been noted by several guideline bodies, which now consider the potential benefits of administrating both BI and a GLP-1 RA $[2,11]$. 
Along with the growing armamentarium of treatment options for T2D, evidence suggests that initiating combination therapy earlier in the course of the disease may be more physiologically appropriate than a stepwise approach [14], further complicating the already challenging task of clinical decision-making in the management of patients with T2D.

Much can be learned about which treatment strategies are most effective in everyday clinical practice through the analysis of large medical record databases, which can provide information on many patients at the point of care [15]. Research Action for Health Network (REACHnet) is a regional US electronic medical records database comprising 13 clinical data research networks funded by the National Patient-Centered Outcomes Research Network (PCORnet). It is a collaborative effort between the Louisiana Public Health Institute, Ochsner Health System, Partnership for Achieving Total Health, Louisiana State University, Pennington Biomedical Research Center, Tulane University, and the University Medical Center, all in Louisiana, as well as Baylor Scott and White Health in Texas, and contains demographic and health information data, including clinical encounters, diagnoses, medications, laboratory data, and procedures on $>5$ million patients of all age, sex, and race groups.

By carrying out a retrospective, observational cohort analysis of data extracted from the REACHnet database, we set out to evaluate whether simultaneous initiation of BI and a GLP-1 RA is more effective at reducing HbA1c than initiating BI and a GLP-1 RA sequentially in patients with T2D who require treatment intensification to achieve glycemic control. This study was planned as a pilot investigation to inform a more robust and comprehensive study using a larger database more representative of the US population in the future.

\section{METHODS}

Patients were included in this retrospective analysis if they had at least one diagnosis of T2D and associated encounter dates between January 2011 and August 2017 (based on data availability); were previously uncontrolled on OADs (HbA1c $\geq 7.0 \%$ ); had no diagnosis of type 1 diabetes or secondary diabetes at any time; had received at least one prescription of $\mathrm{BI}$ after the first recorded T2D diagnosis; had received at least one prescription of GLP-1 RA at the same timepoint (simultaneous initiation) or subsequent (sequential initiation) to their first prescription of $\mathrm{BI}$; had at least one HbA1c laboratory value recorded during the baseline period; had at least two HbA1c laboratory values recorded post-index date; and were $\geq 18$ years of age as of the index date. The date of initiation of BI was defined as the index date, and baseline measurements were defined as those taken within 90 days before and up to the index date. Data of interest, including baseline demographics, disease and clinical characteristics, and HbA1c up to 12 months, were extracted from REACHnet. There was no patient censoring or exclusion based on other treatments received, and no missing data imputation was conducted. All data were censored 1 year after the index date.

With the extracted data, three different treatment sequences were investigated: patients who had simultaneous initiation of BI and GLP1 RA therapy (Cohort 1); patients who were initiated onto BI followed by GLP-1 RA within 90 days (Cohort 2); and patients who were initiated onto BI followed by initiation of GLP-1 RA therapy beyond 90 days (Cohort 3). As this was a pilot study, there was interest in observing whether there was any trend associated with simultaneous initiation, sequential initiation with a short time gap, and sequential initiation with a longer time gap.

The outcomes reported here include the proportion of patients achieving HbA1c $<7.0 \%$ up to 12 months after the index date and change in HbA1c from baseline up to 12 months after the index date. HbA1c data were extracted from the database at baseline and at months 3, 6, 9, and 12 after the patient's index date. If data were not available at each prespecified timepoint, the closest HbA1c laboratory result was taken, provided that it was within 75 days before and within 15 days after each prespecified timepoint. If the prespecified timepoint was the index date, the closest 
preceding laboratory test to the index date (but within the 90 days before and 15 days after) was applied. Each month was rounded to 30 days for HbA1c trend analysis. Only data from patients with valid results at each timepoint were included in the analysis, and no imputation for missing data was made.

Kaplan-Meier analysis was used to estimate the proportion of patients who achieved HbA1c $<7.0 \%$, and pairwise log-rank tests were used to compare outcomes between Cohorts 1 and 2, 1 and 3 , and 2 and 3 . Changes in HbA1c at 3, 6, 9, and 12 months after the index date were estimated and expressed as the mean \pm standard deviation (SD) unless stated otherwise.

Ethics committee approval was not required as this was a retrospective observational study of existing anonymized electronic medical record data that were collected in daily clinical practice. No intervention was implemented on the patients for the purpose of the study, and no patient-identifiable information was used in the study.

\section{RESULTS}

Retrospective analysis of data from REACHnet identified 109 patients who qualified for Cohort 1 (simultaneous initiation of BI and a GLP-1 RA), 301 who qualified for Cohort 2 (initiation of BI followed by sequential initiation of GLP-1 RA within 90 days), and 459 patients who qualified for Cohort 3 (initiation of BI followed by sequential initiation of GLP-1 RA with a gap beyond 90 days). The mean ( \pm SD) length of time to initiation of GLP-1 RA was $36.9 \pm 24.7$ days in Cohort 2 and $330.3 \pm 273.1$ days in Cohort 3 ; the median value was 34 days (1.1 month) and 224 days (7.5 months), respectively. The flowchart of patient cohort selection is illustrated in Fig 1 .

At baseline, the mean $( \pm \mathrm{SD})$ patient age was $51.4 \pm 9.6$ (Cohort 1), $52.5 \pm 10.7$ (Cohort 2), and $53.9 \pm 10.9$ years (Cohort 3 ), and $>80 \%$ of patients had a body mass index $\geq 30 \mathrm{~kg} / \mathrm{m}^{2}$. HbA1c in Cohorts 1, 2, and 3 was $10.3 \pm 2.1 \%$, $10.3 \pm 2.0 \%$, and $10.2 \pm 2.1 \%$, respectively (Table 1). Differences between the cohorts were noted for the proportion of patients with macrovascular disease, chronic kidney disease, and congestive heart failure at baseline. However, no propensity score matching was performed due to the small sample sizes.

The estimated rate of achieving HbA1c $<7.0 \%$ at 12 months was highest in Cohort 1 (33.4\%), followed by Cohort $2(24.5 \%)$, and lowest in Cohort 3 (20.9\%), with a statistically significant difference between Cohort 1 and Cohort $3(p=0.0186)$ and a trend of worsening outcome from Cohort 1 to Cohort 2 to Cohort 3 (Fig. 2a). The greatest mean observed reductions in HbA1c occurred within the first 3 months after the index date and continued to decrease-to a lesser degree-over the next 3 months in Cohorts 1 and 2. Mean $( \pm$ SD) observed HbA1c reductions at 12 months were largest in Cohort $1(-1.7 \pm 2.2 \%)$, followed by Cohort $2(-1.5 \pm 2.5 \%)$, and smallest in Cohort 3 ( $-1.3 \pm 2.4 \%$; Fig. $2 b)$.

\section{DISCUSSION}

A US regional electronic medical records database was used to obtain everyday clinical practice prescribing and $\mathrm{HbA1c}$ data on patients with T2D taking both BI and a GLP-1 RA after inadequate glycemic control on OADs. The purpose of this analysis was to evaluate whether simultaneous initiation of BI and a GLP-1 RA would be more effective at reducing HbA1c than sequential initiation of BI followed by a GLP-1 RA. The results from this pilot investigation show that simultaneous initiation resulted in significantly more patients achieving HbA1c $<7.0 \%$ than sequential initiation with a gap beyond 90 days between commencing $\mathrm{BI}$ and a GLP-1 RA. The likelihood of achieving HbA1c $<7.0 \%$ when GLP-1 RA therapy was started within 90 days after BI therapy was numerically better than when GLP-1 RA therapy was started with a gap of $>90$ days, but worse than with simultaneous initiation. However, the difference did not reach statistical significance when Cohort 2 was compared with either Cohort 1 or Cohort 3, which may be partially due to the small sample sizes. Taken together, our findings indicate that more timely treatment intensification with combination therapy may improve 


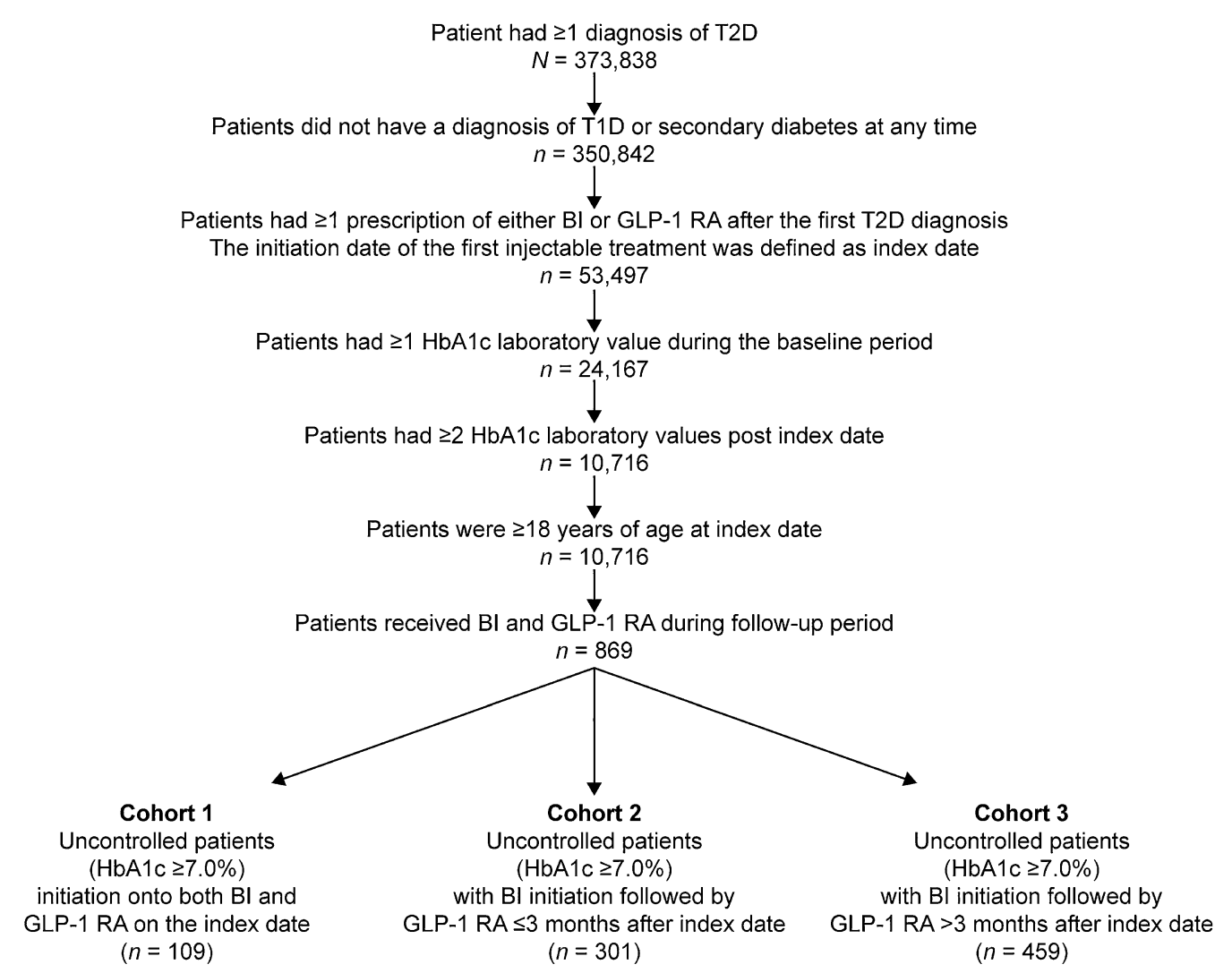

Fig. 1 Flowchart of patient cohort selection. BI Basal insulin, GLP-1 RA glucagon-like peptide-1 receptor agonist, HbAIc glycated hemoglobin, TID type 1 diabetes, $T 2 D$ type 2 diabetes

glycemic control in patients with T2D inadequately controlled on OADs, which is in line with evidence from a previous randomized controlled trial supporting early combination therapies, despite differences in the specific drugs [14]. In this study, approximately $50 \%$ of the patients were initiated onto GLP-1 RA between 90 days and 7.5 months after BI initiation (Cohort 3). However, it is not clear in the current study whether all patients were still taking both injectable medications at 12 months because we did not implement an algorithm to ensure that this was the case for all patients included in the analysis. This will be investigated in a more robust and detailed analysis of a much larger database in our next study.

Although current guidelines advocate the initiation of a GLP-1 RA as the first injectable medication for the intensification of glycemic control in most cases [2], the data collection period in the current study was from 2011 to 2017-before the most recent professional recommendations that are based on the latest plethora of evidence indicating cardiovascular benefits with the GLP-1 RA class of medications [16-18]. Regardless of guidelines, the database is reflective of actual prescribing behavior in everyday clinical practice during the study period. This finding offers an interesting insight into real-world prescribing. Our next planned database analysis will also include another cohort of patients who are initiated onto GLP-1 RAs before BI.

Early treatment intensification is particularly relevant for patients with high HbA1c values, as per the latest ADA's Standards of Medical Care in Diabetes recommendation of combining GLP-1 RA and BI in patients with HbA1c $>10.0 \%$ or HbA1c $>2 \%$ above the target [2]. Our data show that many patients with T2D had a mean baseline HbA1c $>10 \%$ across all 
Table 1 Baseline demographics and clinical, disease, and index date characteristics

\begin{tabular}{|c|c|c|c|}
\hline Characteristics & Cohort $1(n=109)^{\mathrm{a}}$ & Cohort $2(n=301)^{b}$ & Cohort $3(n=459)^{c}$ \\
\hline \multicolumn{4}{|l|}{ Age, years } \\
\hline Mean $\pm \mathrm{SD}$ & $51.4 \pm 9.6$ & $52.5 \pm 10.7$ & $53.9 \pm 10.9$ \\
\hline Median & 52.0 & 53.0 & 55.0 \\
\hline \multicolumn{4}{|l|}{ Sex, $n(\%)$} \\
\hline Male & $62(56.9)$ & $135(44.9)$ & $218(47.5)$ \\
\hline Female & $47(43.1)$ & $166(55.1)$ & $241(52.5)$ \\
\hline \multicolumn{4}{|l|}{ Race, $n(\%)$} \\
\hline White & $72(66.1)$ & $183(60.8)$ & $282(61.4)$ \\
\hline Black or African American & $30(27.5)$ & $114(37.9)$ & $162(35.3)$ \\
\hline Asian & $2(1.8)$ & $1(0.3)$ & $9(2.0)$ \\
\hline Patients with $\geq 1 \mathrm{OAD}, n(\%)^{\mathrm{d}}$ & $63(57.8)$ & $181(60.1)$ & $232(50.5)$ \\
\hline \multicolumn{4}{|l|}{ BMI $\left(\mathrm{kg} / \mathrm{m}^{2}\right)$} \\
\hline Mean $\pm \mathrm{SD}$ & $38.0 \pm 8.8$ & $35.9 \pm 7.0$ & $36.4 \pm 8.3$ \\
\hline Median & 37.0 & 35.0 & 35.0 \\
\hline$<25, n(\%)$ & $3(2.8)$ & $5(1.7)$ & $16(3.7)$ \\
\hline$\geq 25$ and $<30, n(\%)$ & $14(13.0)$ & $39(13.4)$ & $69(15.9)$ \\
\hline$\geq 30, n(\%)$ & $91(84.3)$ & $247(84.9)$ & $349(80.4)$ \\
\hline \multicolumn{4}{|l|}{ HbAlc $(\%)$} \\
\hline Mean $\pm S D$ & $10.3 \pm 2.1$ & $10.3 \pm 2.0$ & $10.2 \pm 2.1$ \\
\hline Median & 10.3 & 10.0 & 9.7 \\
\hline \multicolumn{4}{|l|}{ T2D-related complications, $n(\%)$} \\
\hline Microvascular & $59(54.1)$ & $162(53.8)$ & $200(43.6)$ \\
\hline Macrovascular & $16(14.7)$ & $77(25.6)$ & $135(29.4)$ \\
\hline \multicolumn{4}{|l|}{ Other ${ }^{\mathrm{e}}$} \\
\hline Hypoglycemia & $39(35.8)$ & $109(36.2)$ & $123(26.8)$ \\
\hline Infection & $25(22.9)$ & $77(25.6)$ & $143(31.2)$ \\
\hline \multicolumn{4}{|l|}{ Comorbidities, $n(\%)$} \\
\hline Hypertension & $78(71.6)$ & $236(78.4)$ & $358(78.0)$ \\
\hline Dyslipidemia & $75(68.8)$ & $213(70.8)$ & $330(71.9)$ \\
\hline Chronic kidney disease & $9(8.3)$ & $32(10.6)$ & $53(11.5)$ \\
\hline Depression & $12(11.0)$ & $38(12.6)$ & $47(10.2)$ \\
\hline Congestive heart failure & $3(2.8)$ & $20(6.6)$ & $33(7.2)$ \\
\hline
\end{tabular}


Table 1 continued

\begin{tabular}{llll}
\hline Characteristics & Cohort 1 $(\boldsymbol{n}=\mathbf{1 0 9})^{\mathbf{a}}$ & ${\text { Cohort 2 }(\boldsymbol{n}=\mathbf{3 0 1})^{\mathbf{b}}}^{\mathbf{b}}$ & ${\text { Cohort 3 }(\boldsymbol{n}=\mathbf{4 5 9})^{\mathbf{c}}}^{\text {Index date year, } n(\%)^{\mathrm{f}}}$ \\
2011 & 0 & 0 & \\
2012 & 0 & $1(0.3)$ & $4(0.9)$ \\
2013 & $13(11.9)$ & $67(22.3)$ & $7(1.5)$ \\
2014 & $34(31.2)$ & $78(25.9)$ & $101(22.0)$ \\
2015 & $32(29.4)$ & $76(25.2)$ & $184(40.1)$ \\
2016 & $30(27.5)$ & $73(24.3)$ & $104(22.7)$ \\
2017 & 0 & $6(2.0)$ & $57(12.4)$ \\
\hline
\end{tabular}

Baseline measurements were taken within 90 days before and up to the index date

$B M I$ body mass index, GLP-1 RA glucagon-like peptide-1 receptor agonist, $O A D$ oral antidiabetic drug, $S D$ standard deviation, $T 2 D$ type 2 diabetes

${ }^{\text {a }}$ Cohort 1: simultaneous initiation of basal insulin (BI) and GLP-1 RA

${ }^{b}$ Cohort 2: BI initiation followed by GLP-1 RA within 90 days

${ }^{c}$ Cohort 3: BI initiation followed by GLP-1 RA beyond 90 days

d The percentages of patients on $\geq 1$ OADs could have been underestimated due to the limitations of data collection

e Measured during baseline period and identified using ICD-9/ICD-10 diagnosis codes

${ }^{\mathrm{f}}$ The date of initiation of BI was defined as the index date

three cohorts, which is around the recommended level for combination therapy. Thus, our study results provide another piece of realworld evidence in support of the professional guidelines with respect to the recommended starting HbA1c level for BI + GLP-1 RA combination therapy and also defend the simultaneous approach to therapy, which is not specifically recommended in guidelines except under certain conditions. Furthermore, hypoglycemia and infection rates were high at baseline, consistent with patients who have already been inadequately controlled on OADs and require substantial treatment intensification. In such a population, high morbidity would be anticipated, and it is important to lower the HbA1c level to a near-normal level with potent and safer combinations of BI + GLP-1 RAs instead of multiple insulin injections.

Although we defined an HbA1c of $7 \%$ as the threshold for adequate glycemic control, we recognize that $\mathrm{HbA1c}$ targets should be individualized. A limitation of this real-world database was that it did not collect individual HbA1c targets. Nevertheless, ADA guidelines recommend $<7 \%$ as a reasonable HbA1c goal for many adults [2], and this target was chosen for that reason.

The REACHnet electronic medical records database is a regional PCORnet database, and most of the data obtained were from 2013 to 2016, pre-dating the recent substantial increase in GLP-1 RA use and the more recently marketed GLP-1 RAs, as well as the availability of the fixed-ratio combination therapies. As the choice of treatment was at the discretion of the physician, the lack of randomization may have confounded the comparison between cohorts. Additionally, there were some limitations in the database with respect to collection of information that would have better classified the patients being treated and their response to intensification with injectable agents. We were unable to obtain a reliable estimate of the duration of diabetes at baseline or the details of the type and number of specific OADs patients 

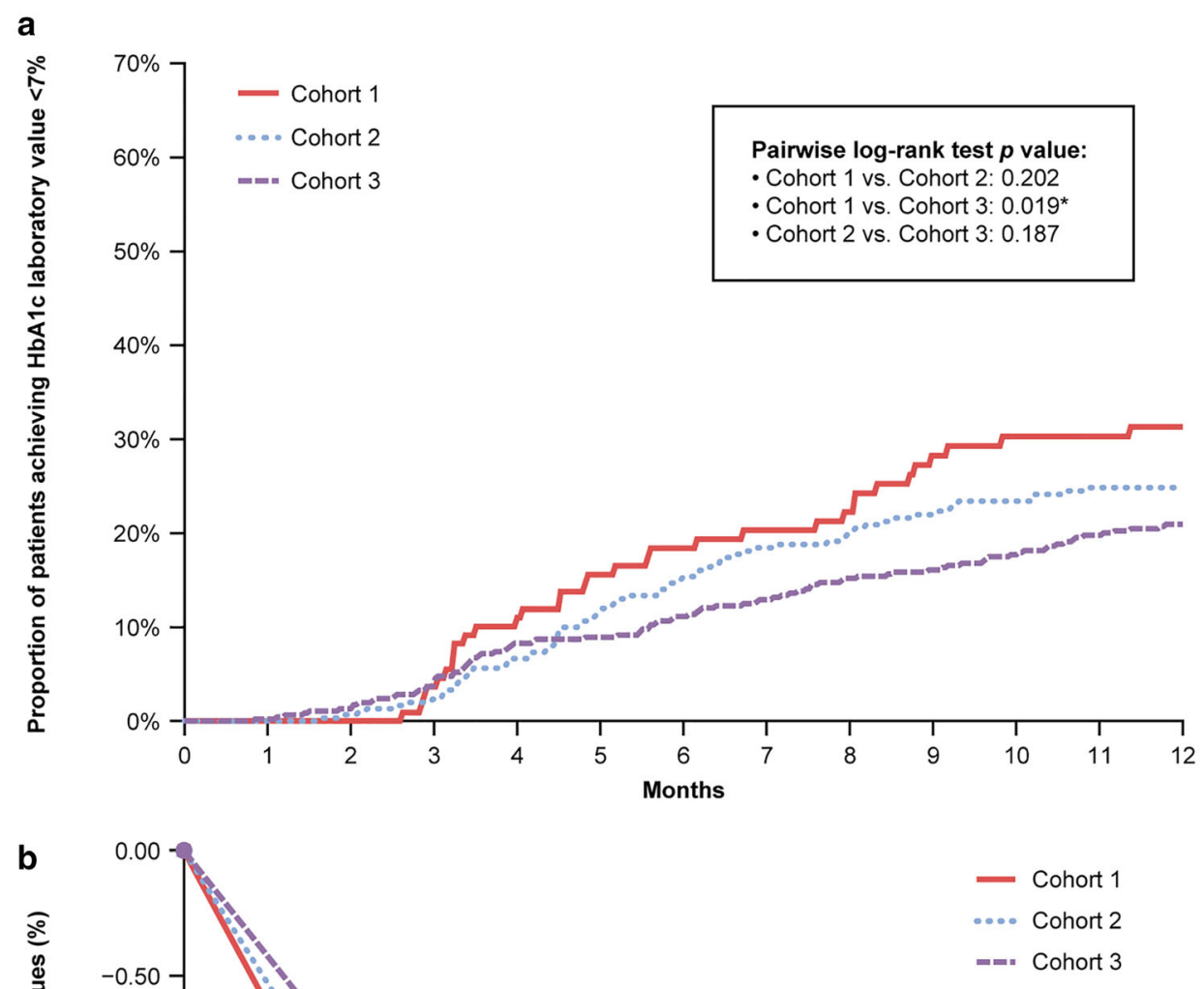

Fig. 2 Proportion of patients achieving $\mathrm{HbAlc}$ value $<7.0 \%$ (a) and mean change in HbAlc values from 3 to 12 months (b). Cohorts 1, 2, and 3 are as defined in Fig. 1 caption. Asterisk indicates significant difference at $p<0.05$. SD standard deviation. Sample size indicates

were taking. There were no feasible records of the titration of the doses of the injectable medications and/or patients' adherence to medication dose during the observation period. Furthermore, information on the impact of treatment intensification on body weight over 
the observation period was not available, and neither was information on whether patients received any concurrent lifestyle education information during that time. These factors will be taken into consideration in our subsequent database study.

The baseline data show that patients in Cohorts 2 and 3 appear to have progressively more comorbidities, including macrovascular disease, chronic kidney disease, and congestive heart failure, than patients in Cohort 1, which could influence the choice of therapy and the target HbA1c. As the sample size of our study population was quite small and this was a realworld retrospective review of clinical practice, our analysis of baseline characteristics was purely descriptive, and it is not possible for us to make any assumptions regarding the impact of baseline characteristics on treatment choice or target HbA1c. Similarly, there are a number of other covariates that could have contributed to the outcome measures seen, but no adjustments were made for these, and the study sample size was too small to carry out multivariate analyses. Both these limitations of the current pilot analysis will be investigated in greater depth in our next study.

It is important to note that the combination of two injectable medications may increase costs and side effects versus either injectable medication alone. This possibility should be weighed against the consequences of poor glycemic control, which could lead to more costly complications and healthcare resource utilizations. Fixed-ratio combination therapies are, however, less expensive than the individual components, and the incidence of gastrointestinal adverse events is less due to gradual titration than with the use of individual components.

Given the limitations of our study, as discussed above, as well as the constraints inherent to retrospective analyses of electronic medical records [19], our findings should be interpreted cautiously. Nevertheless, this study provides some interesting insights into the impact of simultaneous versus sequential initiation of BI and GLP-1 RA for the clinical community. We plan to apply the lessons learned from this study to help improve the methodology of our next study, which will be conducted using a much larger database that is more representative of the US population.

\section{CONCLUSIONS}

Within the constraints of the study limitations, our analysis of a US regional database shows that, among patients with T2D inadequately controlled on OADs, simultaneous initiation of BI and a GLP-1 RA resulted in significantly better glycemic control than sequential initiation of BI and GLP-1 RA with a gap beyond 90 days, suggesting that the longer the gap between the initiation of BI and GLP-1 RA therapy, the poorer the glycemic control outcome. These results provide real-world evidence in line with the latest ADA/EASD recommendation of combination therapy with GLP-1 RA and BI in patients with high HbA1c levels. More robust analysis of a larger database will provide further information on prescribing patterns and health outcomes in the management of patients with T2D in everyday clinical practice.

\section{ACKNOWLEDGEMENTS}

The authors would like to thank Mr John Van Vleet for significant contributions in initiating this project.

Funding. This study, including the design, analysis, and interpretation of data, was funded by Sanofi US Inc., Bridgewater, NJ, USA. The Rapid Service Fee for this article was also funded by Sanofi US Inc., Bridgewater, NJ, USA.

Medical Writing and/or Editorial Assistance. The authors received writing/editorial support in the preparation of this manuscript from Brooke Middlebrook on behalf of Evidence Scientific Solutions, Philadelphia, PA, USA and Philippa Cates, PhD, for Evidence Scientific Solutions, Philadelphia, PA, USA. Support for this assistance was funded by Sanofi US Inc., Bridgewater, NJ, USA. 
Authorship. All named authors meet the International Committee of Medical Journal Editors (ICMJE) criteria for authorship for this article, take responsibility for the integrity of the work as a whole, and have given their approval for this version to be published. All authors had full access to all data in this study, and take complete responsibility for the integrity of the data and accuracy of the data analysis.

Disclosures. Xuejun Victor Peng and Robert Lubwama are employees of Sanofi US, Inc. Rajeev Ayyagari is an employee of Analysis Group Inc., which receives fees from pharmaceutical manufacturers (including Sanofi) for research. Lizheng Shi received research support from Analysis Group, Inc., Blue Cross and Blue Shield of Louisiana, Chiasma, Genentech, and Intuitive Surgical; and received consulting honoraria from Intuitive Surgical. Priscilla Hollander receives research support from Merck \& Co., Pfizer Inc., and Sanofi; and received consulting honoraria from Merck \& Co., Novo Nordisk A/S, Pfizer Inc., and Sanofi. Vivian Fonseca receives research support (to Tulane University) from Bayer and Boehringer Ingelheim; received consulting/lecturing honoraria from Abbott, Asahi, AstraZeneca, Eli Lilly, Intarcia, Novo Nordisk, Sanofi-Aventis, and Takeda; and owns stock/stock options in Amgen, BRAVO4Health, Insulin Algorithms, and Microbiome Technologies. Eboni G. PriceHaywood reports no conflicts of interests associated with her contribution to this manuscript.

Compliance with Ethics Guidelines. Ethics committee approval was not required as this was a retrospective observational study of existing anonymized electronic medical record data that were collected in daily clinical practice. No intervention was implemented on the patients for the purpose of the study, and no patient-identifiable information was used in the study.

Data Availability. The datasets generated during and/or analyzed during the current study are available from the corresponding author on reasonable request.
Open Access. This article is licensed under a Creative Commons Attribution-NonCommercial 4.0 International License, which permits any non-commercial use, sharing, adaptation, distribution and reproduction in any medium or format, as long as you give appropriate credit to the original author(s) and the source, provide a link to the Creative Commons licence, and indicate if changes were made. The images or other third party material in this article are included in the article's Creative Commons licence, unless indicated otherwise in a credit line to the material. If material is not included in the article's Creative Commons licence and your intended use is not permitted by statutory regulation or exceeds the permitted use, you will need to obtain permission directly from the copyright holder. To view a copy of this licence, visit http://creativecommons.org/licenses/by$\mathrm{nc} / 4.0 /$.

\section{REFERENCES}

1. Ali MK, Bullard KM, Saaddine JB, Cowie CC, Imperatore G, Gregg EW. Achievement of goals in U.S. diabetes care, 1999-2010. N Engl J Med. 2013;368:1613-24.

2. American Diabetes Association. Standards of medical care in diabetes-2019. Diabetes Care. 2019;42: S1-194.

3. Hermayer KL, Dake A. Newer oral and noninsulin therapies to treat type 2 diabetes mellitus. Cleve Clin J Med. 2016;83:S18-26.

4. Yki-Järvinen H. Thiazolidinediones. N Engl J Med. 2004;351:1106-18.

5. Lipska KJ, Yao X, Herrin J, et al. Trends in drug utilization, glycemic control, and rates of severe hypoglycemia, 2006-2013. Diabetes Care. 2017;40: 468-75.

6. Khunti K, Nikolajsen A, Thorsted BL, Andersen M, Davies MJ, Paul SK. Clinical inertia with regard to intensifying therapy in people with type 2 diabetes treated with basal insulin. Diabetes Obes Metab. 2016;18:401-9.

7. Khunti K, Wolden ML, Thorsted BL, Andersen M, Davies MJ. Clinical inertia in people with type 2 diabetes: a retrospective cohort study of more than 80,000 people. Diabetes Care. 2013;36:3411-7. 
8. Davies MJ, D'Alessio DA, Fradkin J, et al. Management of hyperglycemia in type 2 diabetes, 2018. A consensus report by the American Diabetes Association (ADA) and the European Association for the Study of Diabetes (EASD). Diabetes Care. 2018;2018(41):2669-701.

9. Nathan DM, Buse JB, Davidson MB, et al. Medical management of hyperglycemia in type 2 diabetes: a consensus algorithm for the initiation and adjustment of therapy: a consensus statement of the American Diabetes Association and the European Association for the Study of Diabetes. Diabetes Care. 2009;32:193-203.

10. Valentine V, Goldman J, Shubrook JH. Rationale for, initiation and titration of the basal insulin/ GLP-1RA fixed-ratio combination products, IDegLira and IGlarLixi, for the management of type 2 diabetes. Diabetes Ther. 2017;8:739-52.

11. Garber AJ, Abrahamson MJ, Barzilay JI, et al. Consensus statement by the American Association of Clinical Endocrinologists and American College of Endocrinology on the comprehensive type 2 diabetes management algorithm-2019 executive summary. Endocr Pract. 2019;25:69-100.

12. Maiorino MI, Chiodini P, Bellastella G, Capuano A, Esposito K, Giugliano D. Insulin and glucagon-like peptide 1 receptor agonist combination therapy in type 2 diabetes: a systematic review and metaanalysis of randomized controlled trials. Diabetes Care. 2017;40:614-24.

13. Moreira RO, Cobas R, Lopes Assis Coelho RC. Combination of basal insulin and GLP-1 receptor agonist: is this the end of basal insulin alone in the treatment of type 2 diabetes? Diabetol Metab Syndr. 2018;10:26.

14. Abdul-Ghani MA, Puckett C, Triplitt C, et al. Initial combination therapy with metformin, pioglitazone and exenatide is more effective than sequential add-on therapy in subjects with new-onset diabetes. Results from the Efficacy and Durability of Initial Combination Therapy for Type 2 Diabetes (EDICT): a randomized trial. Diabetes Obes Metab. 2015;17: 268-75.

15. Martin GS. The essential nature of healthcare databases in critical care medicine. Crit Care. 2008;12:176.

16. Hernandez AF, Green JB, Janmohamed S, et al. Albiglutide and cardiovascular outcomes in patients with type 2 diabetes and cardiovascular disease (Harmony Outcomes): a double-blind, randomised placebo-controlled trial. Lancet. 2018;392:1519-29.

17. Marso SP, Bain SC, Consoli A, et al. Semaglutide and cardiovascular outcomes in patients with type 2 diabetes. N Engl J Med. 2016;375:1834-44.

18. Marso SP, Daniels GH, Brown-Frandsen K, et al. Liraglutide and cardiovascular outcomes in type 2 diabetes. N Engl J Med. 2016;375:311-22.

19. Riley GF. Administrative and claims records as sources of health care cost data. Med Care. 2009;47: S51-5. 